\title{
Learning Process Analysis using Machine Learning Techniques*
}

\author{
LAURA FERNÁNDEZ-ROBLES ${ }^{1}$, HÉCTOR ALAIZ-MORETON ${ }^{2}$, JAVIER ALFONSO-CENDÓN ${ }^{1}$, \\ MANUEL CASTEJÓN-LIMAS ${ }^{1}$ and LUIS PANIZO-ALONSO ${ }^{1}$ \\ ${ }^{1}$ Department of Mechanical, Informatics and Aerospace Engineering and ${ }^{2}$ Department of Electrical, Systems and Automation. \\ Universidad de León. Campus Universitario de Vegazana, 24071, León, Spain. E-mail: \{1.fernandez, hector.moreton, javier.alfonso, \\ manuel.castejon, luis.panizo\}@unileon.es
}

\begin{abstract}
This paper presents a method to evaluate the learning-teaching process using machine learning techniques and the conclusions drawn in an experience on eight courses of very diverse fields. The method is based on data visualization supported by multidimensional scaling. Students' learning behavior can be visually interpreted from the graphical results obtained with this methodology. This proposal allows to identify learning patterns that might either confirm previous assumptions or expose unknown and unexpected knowledge. Instructors who aim at identifying those factors with larger impact on the learning-teaching impact might be potential users of this approach. The results obtained on 426 students prove the usefulness of these techniques as appealing feedback in order to re-adjust the learning-teaching process in consonance with the actual performance of the students. Specifically, a case study about changing the teaching methodology to Blend-Learning by using a content management system through Moodle is presented.
\end{abstract}

Keywords: machine learning; classification; constructivism; Moodle

\section{Introduction}

Analytic methods can shed new light onto the teaching-learning process by providing instructors with useful tools to analyze the performance of students and understand the causes of the observed behaviors [1,2]. Data science is a broad topic with branches that extend into many fields and problem areas [3, 4]. Among the topic areas [5] of greater usefulness, those related with multidimensional scaling, classification and visualization pose special interest for reporting an accurate representation of the status of each and every student enrolled in a specific subject [6].

The bulk of the literature shows how modern approaches to teaching support the idea of getting added benefits from evaluation activities [7, 8], such as improving the learning process itself [9]. Nevertheless, the instructor usually faces the results from the evaluation unarmed or with a set of summarizing tools that lack the expressive power needed to unveil the hidden knowledge that remains captive in the data sets [2]. Instructors can certainly do better than using weighted sums in order to grade their students; and for sure they could use some predictive resources in order to arrange improving actions [10].

Deep analysis of the data collected from a running course [3] may serve well in assessing the impact of developed activities and establishing metrics in order to measure their effectiveness [11] . But it is not only the identification of a problem what matters; knowing what lies ahead is crucial so as to apply corrective measures need they be. In this scenario, our proposal aims to show a simple but effective set of tools that empowers the instructors on their decision making. The usage of analytical visual techniques such as those presented by Gomez-Aguilar [12-15] allows for deeper interpretation of the data collected. Visual approaches ease the monitoring of students' performance while analytical methods enable both instructors and students to determine expected future performance [10].

What follows reports the usage of discriminant analysis on data collected on the students' user experience while implementing a teaching methodology supported by a constructivist learning system. The benefits from using such an analytical and visual approach prove to be decisive by virtue of the interpretation of the results that this technique allows.

This paper focuses on the application of discriminant analysis on a study of the impact of changing the teaching methodology by using a content management system. A questionnaire of 24 questions has been used on 8 courses in a wide range of undergraduate studies. Moodle has been chosen as the platform of choice for supporting Blend-Learning, given that it has been widely adopted by universities worldwide.

The rest of the paper is organized as follows. Section 1 describes the foundations of discriminant analysis and their uses. 


\section{Fundamentals of discriminant analysis}

Discriminant analysis techniques [16], also regarded as supervised classification techniques, work in a scenery in which there exists a finite number $g$ of populations whose probabilistic models of behavior is determined and known [6]. Their purpose is two folded:

- To establish rules that allow to assign the recorded observations to each of the classes. This type of analysis can be regarded as predictive.

- To describe the differences between the populations and define which are the features with better characteristics in the determination or prediction of the class to which an observation belongs. This type of analysis can be regarded as descriptive.

Both analysis can shed light on the data collected. In what follows, both approaches are detailed: first the predictive, using a Bayesian foundation, and second, and algebraic development for the descriptive purpose.

\subsection{Predictive approach to discriminant analysis}

Given $\mathrm{N}$ observations with known values $\left\{\mathrm{x}_{1}, \ldots\right.$, $\left.\mathrm{x}_{\mathrm{N}}\right\}$ recorded on a process in which $\mathrm{g}$ classes of behavior are present $\left\{\mathrm{C}_{1}, \ldots, \mathrm{C}_{\mathrm{g}}\right\}$, the likelihood of a single observation being generated by class $\mathrm{c} \in$ $\left\{\mathrm{C}_{1}, \ldots, \mathrm{C}_{\mathrm{g}}\right\}$ can be easily calculated using Bayes theorem [16] :

$$
P(c \mid x)=\frac{P(c) P(x \mid c)}{P(x)}
$$

Bayes rule, which assigns each observation $\mathrm{x}$ to that class $\mathrm{c}$ with maximum conditional probability $\mathrm{P}(\mathrm{c}$ ) $\mathrm{x})$, minimizes the mean error [18]. Thus, by comparing the value of $\mathrm{P}(\mathrm{c} \mid \mathrm{x})$ given by the different classes, the practitioner can determine to which of them the observation must be assign.

\subsubsection{Quadratic discriminant analysis}

In the determination of the class $\mathrm{c}$ with maximum probability $\mathrm{P}(\mathrm{c} \mid \mathrm{x})$, the denominator in Equation (1) does not contribute any information to the discrimination, being as it is common to all of the classes. Thus, Equation (1) can be simplified by considering only what varies between classes:

$$
P(c \mid x) \propto P(c) P(x \mid c)
$$

Considering now a normal probabilistic model for the behavior of the classes, Equation (2) can be explicitly determined:

$$
P(c \mid x) \propto P(c) \frac{1}{(2 \pi)^{\frac{P}{2}}\left|\Sigma_{c}\right|^{\frac{1}{2}}} e^{-\frac{1}{2}\left(x-\mu_{c}\right)^{T} \Sigma_{c}^{-1}\left(x-\mu_{c}\right)}
$$

Now, instead of finding the maximum of Equation (3) it may be simpler to find the minimum of Equation (4):

$$
\begin{aligned}
-\log P(c \mid x)= & \frac{1}{2}\left(x-\mu_{c}\right)^{T} \Sigma_{c}^{-1}\left(x-\mu_{c}\right) \\
& +\frac{1}{2} \log \left|\Sigma_{c}\right|-\log P(c)+c t e
\end{aligned}
$$

Equation (4) still displays terms which do not vary depending on the class. These can be discarded as well for the sake of simplicity, finally yielding what now can be defined as the quadratic discriminator Qc:

$$
\begin{aligned}
Q_{c}= & \frac{1}{2}\left(x-\mu_{c}\right)^{T} \Sigma_{c}^{-1}\left(x-\mu_{c}\right) \\
& +\frac{1}{2} \log \left|\Sigma_{c}\right|-\log P(c)
\end{aligned}
$$

Thus, in order to establish which class originated a given sample, the class with lower Qc must be chosen.

Further study on the definition of Qc reveals the presence of $\mathrm{D}(\mathrm{x} \mid \Theta \mathrm{c})$, the square of the Mahalanobis distance of $x$ to the center of the multivariate normal distribution with parameters $\Theta \mathrm{c}=\{\mu \mathrm{c}, \Sigma \mathrm{c}\}$ :

$$
Q_{c}=\frac{1}{2} D^{2}\left(x \mid \Theta_{c}\right)+\frac{1}{2} \log \left|\Sigma_{c}\right|-\log P(c)
$$

This implies that the frontiers separating the domains of prevalence for each class will be quadratic. Fig. 1 shows such a map where three random populations have been used for illustrative purposes.

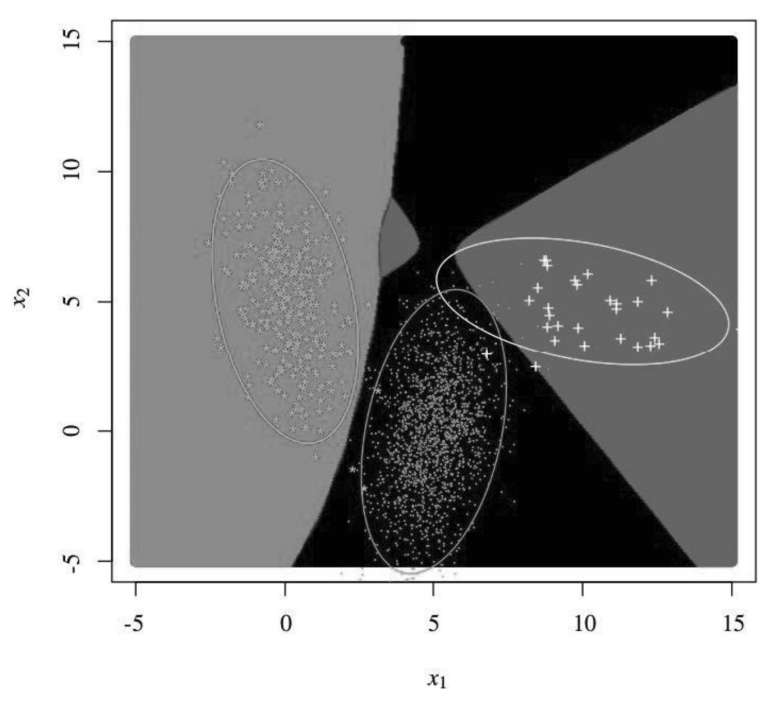

Fig. 1. Quadratic discriminant map for three random classes. 


\subsubsection{Linear discriminant analysis}

Equation (6) can be further developed. Considering that $\mathrm{x}^{\mathrm{T}} \Sigma_{\mathrm{c}^{-1}} \mu_{\mathrm{c}}$ is a scalar and, thus, equivalent to its transpose $\mu^{\mathrm{T}} \Sigma_{\mathrm{c}^{-1}} \mathrm{X}$, gives:

$$
\begin{aligned}
Q_{c}= & \frac{1}{2} x^{T} \Sigma_{c}^{-1} x-x^{T} \Sigma_{c}^{-1} \mu_{c}+\frac{1}{2} \mu_{c}^{T} \Sigma_{c}^{-1} \mu_{c} \\
& +\frac{1}{2} \log \left|\Sigma_{c}\right| P-\log P(c)
\end{aligned}
$$

The term $\mathrm{x}^{T} \Sigma_{\mathrm{c}}^{-1} \mathrm{x}$ is responsible for the quadratic nature of Qc. Assuming a set of classes sharing a common variance-covariance matrix $\Sigma c=\Sigma$, this quadratic term can be discarded for being common to all of the classes, and a new linear discriminator, Lc, can be used instead:

$$
L_{c}=x^{T} \Sigma^{-1} \mu_{c}+\frac{1}{2} \mu_{c}^{T} \Sigma^{-1} \mu_{c}-\log P(c)
$$

Now the decision frontiers are linear and easier to determine. For two given classes, a simple discriminant expression such as $\mathrm{L} 2-\mathrm{L} 1=0$ is as simple as it gets:

$$
L_{2}-L_{1}=x^{T}\left(\mu_{2}-\mu_{1}\right)+\kappa_{2}-\kappa_{1}
$$

Fig. 2 shows the linear discriminant map for three random population, such as those used in Fig. 1, for illustrative purposes. When compared, linear discriminant analysis has discarded the fine nuances displayed by quadratic discriminant analysis.

For predictive purposes, it might not be that interesting to use Lc instead of Qc; but linear discriminant analysis poses unique benefits for descriptive purposes, as it will be shown below.

\subsection{Descriptive approach to discriminant analysis}

The focus now will be to determine linear combinations such as:

$$
\begin{gathered}
y_{1}=\pi_{11} X_{1}+\cdots+\pi_{1 p} X_{p} \\
y_{2}=\pi_{21} X_{1}+\cdots+\pi_{2 p} X_{p} \\
\cdots \\
y_{p}=\pi_{p 1} X_{1}+\cdots+\pi_{p p} X_{p}
\end{gathered}
$$

so that the coordinates of an observation $\mathrm{x}$ will be expressed in a new algebraic base whose components are traditionally named discriminant functions.

In order to determine the coefficients of this base of discriminant functions, two goals are established:

1. The new base must provide maximum separation between the different classes.

2. The classes will be internally as compact as possible.

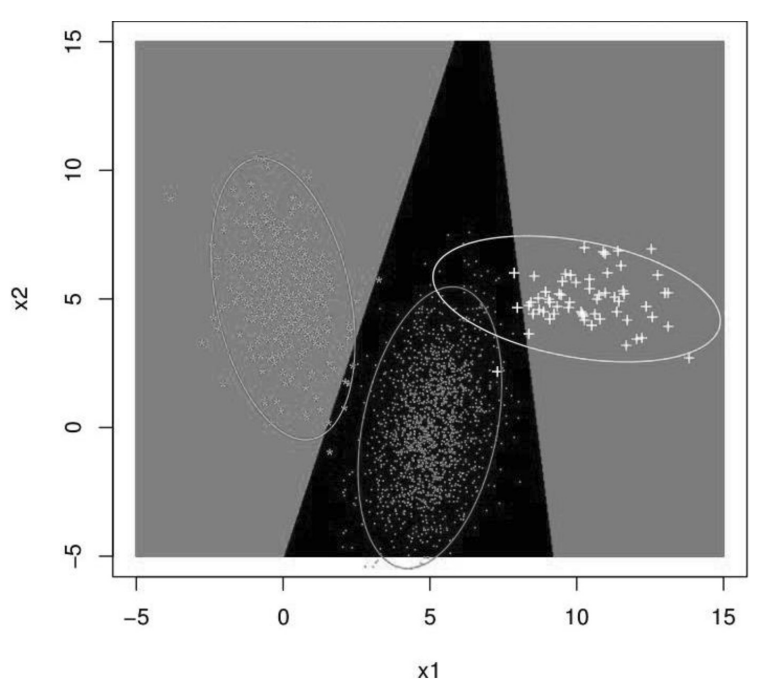

Fig. 2. Linear discriminant map for three random classes.

Two assumptions are made in what follows:

- The non-degenerated multivariate normality of the classes

- Homoscedasticity of the variance-covariance matrices $\left(\Sigma_{1}=\Sigma_{2}=\ldots=\Sigma_{\mathrm{g}}\right)$

Many authors have claimed the robustness of the method against deviations from these two assumptions. Generally speaking, if the number of samples is large and the groups are formed by a similar number of samples the robustness increases. Nevertheless, it seems sensible to delve on the consequences of not meeting these assumptions and the technique available to identify this situation.

\subsubsection{Non-degenerated multivariate normality}

As long as the distribution of the variables does not deviate from multivariate normality, good quality results can be expected [19].

The most important consequences will be found on the hypothesis tests, as they test concrete parameters over theoretical distribution models. The lack of normality may wind up from the presence of an outlier or the lack of symmetry on the distribution shape. Generally speaking, if the data set has a large number of observations, the impact of this lack will not be especially important. In that case, a deeper analysis of those observations lying closer to the decision frontiers would suffice.

The distributions must be non-degenerated, that is, the variance-covariance matrices cannot have null autovalues. This situation can appear both when two variables are highly correlated and contain similar information (singularity), and when one of the variables can be expressed as a linear combination of the others (multicollinearity). In both 
cases the variance-covariance matrix does not have complete rank and thus cannot be inversed.

In large data set, the likelihood of either perfect singularity or multicollinearity is negligible. Nevertheless, high correlation values imply ill conditioned variance-covariance matrices and unstable inversions. This situation is not one to worry too much as the solution is pretty simple just by applying stepwise variable selection techniques and sequentially adding variables to the discrimination.

\subsubsection{Homoscedasticity}

This assumption is usually the one harder to meet. To be identical, the variance-covariance matrices must be identical term by term, what for a couple of symmetrical matrices means $\frac{p(p-1)}{2}$ identical elements. Hence, this a very severe assumption.

Nevertheless, for large samples, or groups with similar number of members, the robustness of the method is highly regarded.

In those cases, where the deviation from the assumption is important, the observations tend to be assigned to those groups with large volume. In this case, if the number of samples is small, the linear discriminant analysis might provide satisfactory results. If the quality of the prediction is compromised, quadratic discriminant analysis must be considered.

Finally, it can be proposed that the best check to test every particular case comes from verifying the quality of the results on labelled data, thus knowing the impact of the deviations from the assumptions.

The determination of the discriminant functions requires the definitions of the following matrices:

$$
\begin{gathered}
T=W+B \\
B=\frac{(G M-\bar{x})^{T}(G M-\bar{x})}{g-1} \\
W=\frac{(X-G M)^{T}(X-G M)}{N-g}
\end{gathered}
$$

where $M=\left(\bar{x}_{1}, \ldots, \bar{x}_{g}\right)^{T}$ is a matrix that comprises the mean vectors of the different classes and $\mathrm{G}$ is a matrix with label, whose elements $g_{i j}$ will be 1 if $x_{i} \in$ $\mathrm{C}_{\mathrm{j}}$ or 0 otherwise.

This way we consider in matrix $\mathrm{W}$ the variance of the observation $\mathrm{x}_{\mathrm{i}}$ relative to the mean $\mathrm{x}_{\mathrm{j}}$ of class $\mathrm{C}_{\mathrm{j}}$.; while reflecting in matrix $B$ the variance of $x_{j}$ relative to the global centroid.

For a given linear combination $\sum_{i=1}^{p} v_{i} X_{i}$, the variance associated to the direction given by $\mathrm{v}$ will be $\sigma^{2}(\mathrm{v})=\mathrm{v}^{\mathrm{T}} \mathrm{Tv}$, that can be further decomposed in the intra-classes variance $\mathrm{v}^{\mathrm{T}} \mathrm{Wv}$ and the inter-classes variance $\mathrm{v}^{\mathrm{T}} \mathrm{Bv}$.
Considering

$$
\lambda=\frac{v^{T} B v}{v^{T} W v}
$$

the $\lambda$ parameter provides high values to those directions with large inter-classes variance and small intra-classes variance; while providing small values for those directions that overlap the classes and where the internal geometry is inflated.

Thence, the most interesting direction can be obtained by optimizing:

$$
\frac{\delta \lambda}{\delta v}=\frac{2\left[B v\left(v^{T} W v\right)-\left(v^{T} B v\right) \lambda W v\right]}{\left(v^{T} W v\right)^{2}}
$$

Assuming that $\mathrm{W}$ is not singular (non-degenerated case) this expression can be simplified:

$$
\frac{\delta \lambda}{\delta v}=\frac{2(B v-\lambda W v)}{v^{T} W v}
$$

By restricting $\frac{\delta \lambda}{\delta v}=0$, the numerator of Equation (16) must be zero then:

$$
(B-\lambda W) v=0
$$

which yields

$$
\left(W^{-1} B=\lambda I\right) v=1
$$

The solutions to (18) are the roots of $\left|\mathrm{W}^{-1} \mathrm{~B}-\lambda \mathrm{I}\right|=0$, which is no other than the characteristic polynomial of matrix $\mathrm{W}^{-1} \mathrm{~B}$.

The problem now is stated in a known form, as the diagonalization of $\mathrm{W}^{-1} \mathrm{~B}$ and the interpretation of the results in a similar manner as that of the principal components analysis. The autovalues obtained will indicate the discriminant power of the functions obtained, the autovectors will be similarly analyzed to reveal the impact of the original variables on the discrimination.

Most commonly, these functions are sorted according to their discriminant power.

\subsection{Application to the learning context}

To show a possible application where techniques such as discriminant analysis can be employed, we elaborate in what follows on a study of the impact of changing the teaching methodology by using a content management system. Moodle has been chosen as the platform of choice for supporting Blend-Learning, given that it has been widely adopted by universities worldwide.

Instructors considered as very positive factors the ease of use of the Moodle environment, both for students and faculty, with no prior programming 
requirements and the capability of recycling the resources created on the courses.

Moodle is based on the pedagogical constructivism principles [20], that is, the learning process is empowered when done in a cooperative manner; which is one of the main considerations for those on the European Higher Education Area

Such a tool makes easier for the instructors to know which student are highly involved on the process and those that not, help them in following the self-assessment of their learning and giving them feedback.

As far as the students are concerned, the Moodle environment has provided an increase in the motivation of the student, since the learning process is focus on the student; it provides easy management of schedules, deadlines, evaluation and feedback, as well as easy access to formative resources on demand.

The resolution of problems and answers to learning doubts out of office hours, being it through virtual interviews or face to face meetings with instructors and colleagues has also been considered as positive factors, along with the increase in the review of bibliographical sources.

So as to evaluate the particular results of this approach versus those obtained via traditional channels, a questionnaire of 24 questions has been designed. This questionnaire has been used on 8 courses in very different studies, ranging from engineering to nursing studies or biotechnology, among others. A global number of 426 answers were collected and analyzed using discriminant analysis.

\section{Initial results}

We obtained a dendrogram by applying the classification algorithm in the obtained data from the inquiry. The dendrogram suggests the existence of two main classes that, if a more detailed description is needed can be broken down to three principal submodes. The values of the hierarchical index significantly make a difference these two configurations from the rest.

In regard to two considered classes, Fig. 3 shows the projection of the Lineal Discriminant Analysis (henceforth LDA) of the two identified classes. It is clearly visible the presence of the two considered classes, even though the separation between them is not perfect and an overlap region is visible. This overlap can be due to the significativity of a third submode of behavior, as it is later proved.

For three considered classes, Fig. 4 shows the average profiles that represent each class by considering a cut of the dendrogram in three branches. In the next section, we provide a detailed analysis of the characteristics of each class with respect to the questions asked and the responses provided. Class 1 comprehends the responses of 173 students, class 2 contains 158 and class 3, 95 .

\section{Discussion}

The inquiry consists of 24 questions. Regarding the age profile, class 1 presents a higher dispersion than classes 2 and 3 . The age distribution of class 1 follows a smoother and less sharp Gauss curve than the Gauss curves of classes 2 and 3. In classes 2 and 3 the distribution axe of their Gauss curves is centered at age $19(44,36 \%$ of the population is 19 for class 2 and $57,89 \%$ for class 3 ). Class 3 is centered at an age between 19 and 20, being 78,95\% of the population in that range and with less significative percentages in the rest of ages. Therefore, class 3 is made up of younger students who are closer and present lower rejection to new information and communication technologies, according to
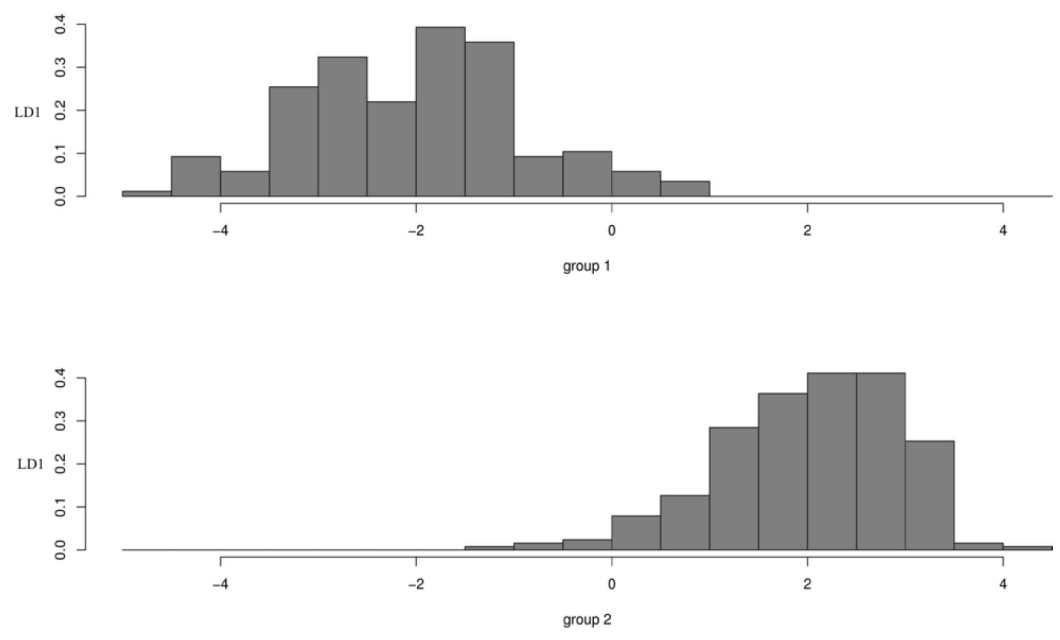

Fig. 3. Projection LDA of the two main classes. 


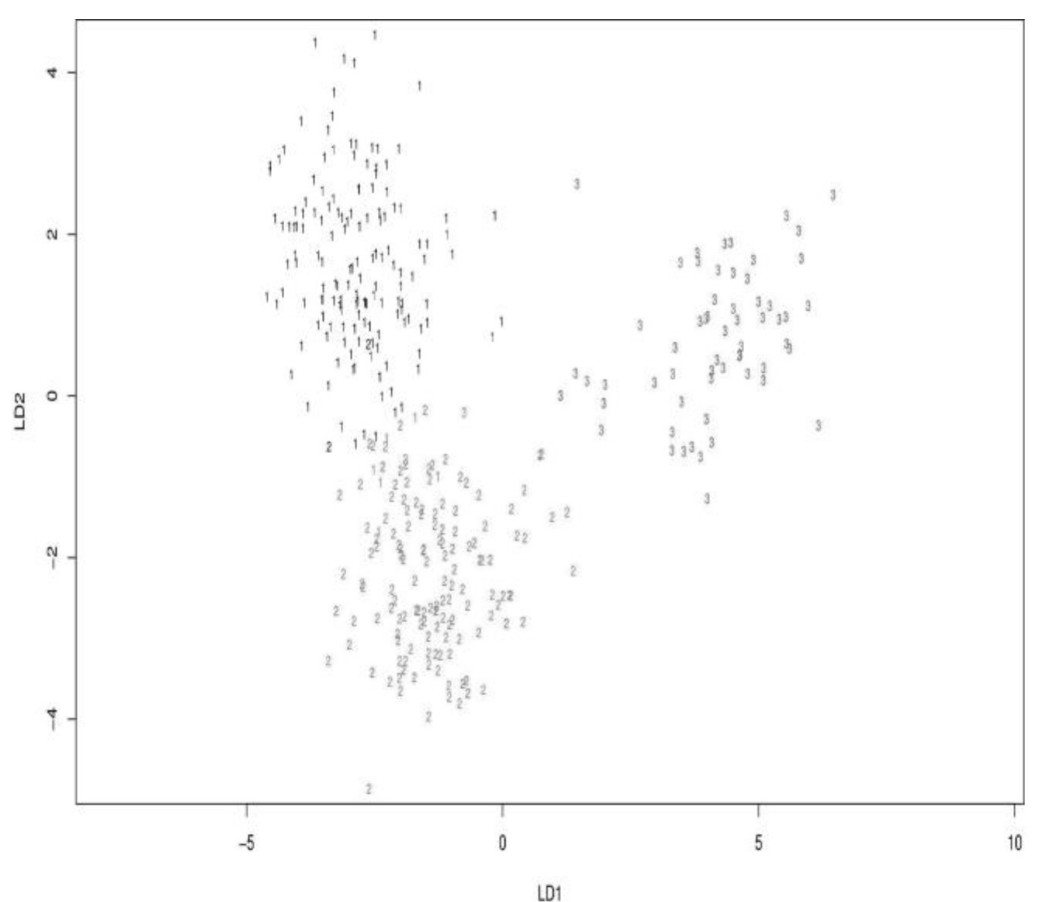

Fig. 4. Average profiles that represent the 3 classes by considering a cut of the dendrogram. Each class is represented with an identifying number.

recent research [21-23]. Researchers pointed out that young people did not meet the so-called digital divide. The closeness and ability to new information and communication technologies made us suppose a higher predisposition to use Moodle as a friendly tool in the development of the university work of class 3 .

With respect to the commitment in time, it seems clear that class 3 employs a higher percentage of weekly time to work in the course with this method, followed by class 1 and then class 2 . This may indicate that using a methodology using virtual classroom Moodle, based on tasks and a more continuous monitoring of these tasks, demands a certain level of dedication to the course. This has been expressed in items 22 and 23 of the inquiry in which students were asked about the most positive and negative of this methodology. Some survey respondent valued very positively this methodology, stating, "it allows me to work in a more organized way and without a great load of work at the end" or "it is very encouraging, because we can see the progress of our effort with an immediate assessment of the performed tasks". On the contrary, some answers were more critical mentioning, "the time to perform an assignment is tight" or "we have many tasks to perform in a very tight time". These two types of divergent responses indicate that the valuation in terms of time and effort dedicated to these tasks is essentially related with the learning style and with the motivation factors and effort that subjectively influence them.
We can conclude, therefore, that even most of the students pointed out that training is not required for the management of the virtual platform, it would be appropriate to provide some concepts and operating instructions for the $5-15 \%$ of students that demand a previous training. It is a low but notable percentage that justifies a small training. A higher level of training should be given to students of classes 2 and 3, who are the youngest and less accustomed to use these learning tools.

Regarding the question about the teacher ability to react or respond, class 1 considers that the teacher response was very fast or fast $(75 \%)$. Also, class 2 $(51 \%)$. However, class 3 , which presented more difficulties in the management of the learning platform, thinks that the teacher response was slow $(28 \%)$ or do not know how this response was $(53 \%)$, maybe because they did not try to ask doubts or questions to the teacher. This is an evidence of the "apathy" or "ignorance" in the use of the platform that class 3 presents.

With respect to the clarity of the proposed tasks, classes 1 and 2 appreciate good clarity and they were reasonably satisfied ( $95 \%$ for class 1 and $87 \%$ for class 2). For class 3 the system is more confusing, only $25 \%$ of the students are satisfied while $54 \%$ are disoriented with respect what they need to do. There is again a manifest insecurity to confront the performance of tasks using the virtual environment Moodle. Students of class 3 are disoriented or lost in this education system because they do not clearly understand what they need to do. This system is 
probably too novel, generating distrust and rejection of the student towards the system. Consequently, even though class 3 represents the minority of the student body, in future it will be necessary to pay attention to such diversity, specially to the orientation and monitoring of these students.

As to the rhythm of the classes, there are essential differences in the profile of the three types of identified behavior. All 3 classes comprehend responses embracing the full spectrum of diverse possibilities, clearly class 1 focus its responses in the most favorable area with regard to rhythm. Class 2 follows closely class 1 , but with lower rate of response "Very.Appropriate.Rhythm". Class 3 finds more difficulties with the imposed rhythm of learning, with a high number of responses in the unfavorable spectrum.

For the question, would you have preferred to do these practical activities in a presence-based modality? Class 1 is clearly in favor of the system in the majority of cases whereas classes 2 and 3 have mixed feelings and most of the opinions say that in some cases it is better a presence-based system. It is also remarkable that in classes 2 and 3 there is a small portion of students (11 to $15 \%$ ) who do not know what to answer.

We analyze now the consideration that the platform allows to pursue your own personal learning system, adapting the education to your timetable and location availabilities. We can conclude that the three classes believe, to a different extend, in the advantages of this system with respect to the availability. There is a partial exception in class 3 in which there is an important level of ignorance.

Another question assessed if the student possibilities of consulting bibliographic material using electronic means, audiovisual media, email and Internet nets. We conclude that class 1 agrees or highly agrees with this statement. Class 2 is less positive but yet agrees. And responses of class 3 are very dispersed between agree and disagree and ignorance.

It is surprising that class 3 , with a younger population and admitting having spent more time, is the one that perceives a lower increase of selfcontrol. The reason might be that they have good enough feelings about their personal effort regardless the learning system. On the contrary, classes 1 and 2, which declare to dedicate less hours to learn, consider very appropriate this system as a tool to improve their self-control feelings.

$77 \%$ and $87 \%$ of the students in class 1 and 2 , respectively, agree or highly agree with the suitability of the system. Surprisingly, in spite of the global negativity of class 3 towards this system in other questions of the inquiry, $53 \%$ of the students agree with the use of this system and only $20 \%$ think that it is not useful. All in all, the vast majority of the survey respondents conclude that the use of this system is very appropriate, regardless the utility their own experience with it.

In relation to the degree of satisfaction with the system, there are big differences among the three classes. $85.49 \%$ of class 1 are satisfied or highly satisfied about their participation in a course with this methodology. On the other hand, $35.53 \%$ of class 3 is not satisfied with their participation in courses with this methodology and a $30.26 \%$ do not know if they are satisfied or not. Therefore, $66.79 \%$ of students in class 3 did not get a good experience with this methodology.

Less than $10 \%$ of the students do not agree about the repetition of the experience, which means to have more courses with this methodology. This rate varies among classes: class 1 presents a higher and clearer interest in continuing with this methodology, $78.82 \%$ agrees or highly agrees with it. Class 2 shows a high interest $(61.06 \%$ ), but $30.97 \%$ of the students present doubts about their preference to get courses with this methodology and mark "I do not know". Class 3 is more reluctant with $23.68 \%$ of students that reject to participate again in this kind of experiences and $31.58 \%$ of students that do not know if they would like to repeat this methodology in other courses. Students of class 3 are less satisfied with the system.

Students were asked to enumerate three reasons that they considered as the main difficulties for their performance under this learning experience through Internet. The most repeated reason was time. Students considered they had few time to carry out all the activities that they were asked for.

Many students of class 3 pointed out the lack of communication with the teacher as another factor that made difficult their correct performance $(52.63 \%)$. This is a hint of the difficulties that these students have with regard to independent learning because they are more committed when confronting blended-learning tasks.

The third reason most significant in class 3 is that the course was too heavy, with too many contents. Class 2 also pointed out this fact $(34.51 \%)$. This indicates the perception of the difficulty level of the courses is very different between teachers and students and we should make an effort to adapt the courses to a difficulty level more suitable with respect to the learning level of all students.

The fact that some students did not have Internet access at home has been pointed out by $34.32 \%$ of the survey respondents. They needed to take advantage of the time they were at University without classes or move from their houses to perform the tasks. 
Finally, we would like to highlight that only a $6.23 \%$ of students have shown to not understand Moodle platform as a significant element of difficulty for their performance. We conclude that Moodle is a great means of learning at University range.

\section{Conclusions}

We have reported the results from the usage of a visual and analytical technique on the analysis of data collected from students on the experience of using a new tool in the classroom.

The results obtained by using such technique, discriminant analysis in this case, suggest the presence of three clearly separated classes. The profiles obtained show that students belonging to one of the classes display an outstanding predisposition to the use of servers such as Moodle. Students from another class, with more moderate responses, are also comfortable and interested in this kind of systems. The total number of students in these two classes are a $78 \%$ of the total survey respondents. Students from a third class - $22 \%$ of the total survey respondents - answer in a very critical way most of the questions, but they admit certain interest for this kind of education and are predisposed to continue the experiment.

Machine learning tools, such as discriminant analysis have proven useful in providing insights on the performance of students and the causes that drive them to enhance their results. Instructors can take advantage of such tools to monitor the learning process and to identify areas in need of extra efforts.

\section{References}

1. N. D. Tran, Reconceptualisation of approaches to teaching evaluation in higher education, Issues in Educational Research, 25(1), 2015, pp. 50-61.

2. C. Dennison, R. Butz, F. Shawn and J. Carey, Comparison of Student Evaluation of Teaching Results when Stratified by Protocol, Course Content, and Course Structure, International Journal of Engineering Education, 31, 2015, pp. 14761490.

3. S. Fosso Wamba, S. Akter, A. Edwards, G. Chopin and D. Gnanzou, How "big data" can make big impact: Findings from a systematic review and a longitudinal case study, International Journal of Production Economics, 165, 2015, pp. 234-246.

4. Y. Park, J. H. Yu and I. H. Jo, Clustering blended learning courses by online behavior data case study in a Korean higher education institute, Internet and Higher Education, 29, 2016, pp. 1-11.

5. D. Kao Ming, C. Hao, M. A. Livingston, T. Wischgoll and
D.Lid, Visualization and Data Analysis, Visualization and Data Analysis Proc. of SPIE-IS\&T Electronic Imaging Proceedings of SPIE-IS\&T Electronic Imaging, SPIE Article CID Number. 9397, 2015.

6. O. E. Tsivitanidou and C. P. Constantinou, A study of students' heuristics and strategy patterns in web-based reciprocal peer assessment for science learning, Internet and Higher Education, 29, 2016, pp. 12-22.

7. D. Nicol, A. Thomson and C. Breslin, Rethinking feedback practices in higher education: a peer review perspective. Assessment \& Evaluation in Higher Education, 39(1), 2014, pp. $102-122$.

8. Z. Stojanov and D. Dobrilovic, Qualitative Evaluation of Software Maintenance Services Integrated in a Virtual Learning Environment, International Journal of Engineering Education, 32, 2016, pp. 790-803.

9. L. Greer and M. Mark, Evaluation Methods for Intelligent Tutoring Systems Revisited, International Journal of Artificial Intelligence in Education, 26(1), 2016, pp. 387-392.

10. J. W. You, Identifying significant indicators using LMS data to predict course achievement in online learning, Internet and Higher Education, 29, 2016, pp. 23-30.

11. D. Mishra, S. Ostrovska and T. Hacaloglu, Assessing Team Work in Engineering Projects, International Journal of Engineering Education, 31, 2015, pp. 627-634.

12. D. A. Gomez Aguilar, M. A. Conde-González, R. Theron and F. J. Garcia-Penalvo, Reveling the Evolution of Semantic Content Through Visual Analysis, Proceedings of the 2011 IEEE 11th International Conference on Advanced Learning Technologies (Washington, DC, USA), 2011, pp. 450-454.

13. D. A. Gomez Aguilar, A. Hernández-García, F. J. GarcíaPeñalvo and R. Therón, Computers in Human Behavior Tap into visual analysis of customization of grouping of activities in eLearning, 47, 2015, pp. 60-67.

14. D. A. Gomez Aguilar, R. Therón and F. J Peñalvo, Reveal the relationships among students participation and their outcomes on e-learning environments: case study, 2013 IEEE 13th International Conference on Advanced Learning Technologies, 2013, pp. 443-447.

15. D. A. Gómez Aguilar, H. van Zuylen and F. J. GarcíaPeñalvo, Semantic Spiral Timelines Used as Support for eLearning, Journal of Universal Computer Science, 15(7), 2009, pp. 1526-1545.

16. T. Jombart, S. Devillard and F. Balloux, Discriminant analysis of principal components: a new method for the analysis of genetically structured populations, BMC Genetics, 11(1), 2010, pp. 1-15.

17. C. Fraley and A. E. Raftery, Model-based clustering, discriminant analysis, and density estimation, Journal of the American Statistical Association, 97(458), 2002, pp. 611-631.

18. W. N. Venables and B. D. Ripley, Modern Applied Statistics With S, Statistics and Computing, Springer, 2002.

19. C. P. López, Minería de datos: técnicas y herramientas, Paraninfo Cengage Learning, 2007.

20. moodle, https://moodle.org/, accessed 10 October 2017.

21. E. D. Cassidy, A. Colmenares, G. Jones, T. Manolovitz, L. Shen and S. Vieira, Higher Education and Emerging Technologies: Shifting Trends in Student Usage, The Journal of Academic Librarianship, 40(2), 2014, pp. 124-133.

22. P. I. Cerretani, E. B. Iturrioz and P. B. Garay, Use of information and communications technology, academic performance and psychosocial distress in university students, Computers in Human Behavior, 56, 2016, pp. 119-126.

23. J. Lu, Q. Hao and M. Jing, Consuming, sharing, and creating content: How young students use new social media in and outside school, Computers in Human Behavior, 64, 2016, pp. $55-64$.

Laura Fernández Robles received the BSc (2009) degree in Industrial Engineering from University of León where she graduated with honours (first class). In 2011, she received the MSc degree in Intelligent Systems in Engineering at University of León and in 2016 the PhD degree from University of León, Spain and from University of Groningen, the Netherlands. In the present, she is an assistant lecturer and researcher at University of León. Her current research interests include computer vision, pattern recognition and machine learning in different fields of application. 
Héctor Alaiz Moretón received his degree in Computer Science, performing the final project at Dublin Institute of Technology, in 2003. He received his PhD in Information Technologies in 2008 (University of León). He has worked like a lecturer since 2005 at the School of Engineering at the University of León. His research interests include knowledge engineering, networks communication and security. He has several works published in international conferences, as well as books and scientific papers in peer review journals. He has been member of scientific committees in conferences. He has headed several $\mathrm{PhD}$ Thesis and research projects.

Javier Alfonso Cendón is Associate Professor in Project Management at Universidad de Leon. He is PhD in Engineering by the University of Leon. He has many specialized courses in the field of engineering and communication technologies. His research interests include project management issues, business intelligence, data mining, bioinformatics and machine learning. He has several publications in the field of ICT. He serves as reviewer for different peer-reviewed journals.

Manuel Castejón Limas is Associate Professor at Universidad de León where he lectures Project Management in Industrial Engineering undergraduate and master's degrees. His research interests are focused on applying data-driven approaches to quality optimisation and processes modelling. His past experiences in applying optimisation techniques to industry related problems in the steel-making area lead to the implementation of robust predictive models on hot dip galvanising lines and steel annealing furnaces as well as predictive systems for steel coils cleaning systems and the determination of mechanical properties of the final product. In the automotive industry, he has applied these techniques to equally improve the quality of rubber extrusion processes by the deployment of a data-driven manufacturing support system.

Luis Panizo Alonso received the BSc (1980) degree in Telecommunication Engineering from Universidad Politécnica de Madrid and received the MSc degree in Cybernetics Engineering and the PhD degree from University of León, Spain. He has several works published in international conferences, as well as books and scientific papers. He has headed several European research projects and his research interests include electronic voting and cybersecurity. In the present, he is an Associate Professor and researcher at University of León. 